\title{
Development and physicochemical characterization of a new grass pea (Lathyrus sativus L.) miso
}

\author{
Rafaela Santos, ${ }^{*} \odot$ Ana Mansidão, Mariana Mota, $\odot$ Anabela Raymundo $\odot$ and \\ Catarina Prista
}

\begin{abstract}
BACKGROUND: Western consumers interest in Eastern fermented foods has been growing, due to their nutritional and healthy properties. In this study, new sweet misos and salty misos were produced using grass pea (Lathyrus sativus L.) - traditional Portuguese legume from local producers - to promote its consumption and preservation. The evolution of the new misos was evaluated in comparison to traditional miso (made from soybean), through analysis of the chemical composition, colour, texture and linear viscoelastic behaviour.

RESULTS: Throughout the fermentation process, the ascorbic acid and phenolic compounds content - with important nutritional value - increased in all misos, mainly in misos produced using grass pea, besides, grass pea sweet miso presented the fastest evolution and darkest colour. The texture parameters (firmness and adhesiveness) of misos decreased over time: grass pea sweet miso showed the highest firmness reduction (51.63 $\mathrm{N}$ to $6.52 \mathrm{~N}$ ) and soybean sweet miso the highest adhesiveness reduction (27.76 $\mathrm{N}$ to $3.11 \mathrm{~N})$. Viscoelastic moduli also decreased, reflecting a reduction in the degree of internal structuring for all misos. However, grass pea misos presented more structured internal systems with faster maturation kinetics than soybean misos, for which stabilization started earlier.
\end{abstract}

CONCLUSION: Two innovative misos were developed from grass pea. After 4 months, the texture parameters and viscoelastic moduli for grass pea misos, were similar to the control misos made from soybean, showing that grass pea can be used as a raw material to produce a sustainable miso with potentially healthy properties.

(c) 2020 Society of Chemical Industry

Keywords: miso; grass pea; chemical composition; colour; texture; linear viscoelastic behaviour

\section{INTRODUCTION}

In the last decade, the concern of Western consumers regarding healthy and environmentally sustainable foods ${ }^{1}$ has contributed to an increasing interest in Eastern legume fermented foods as a source of healthy and eco-friendly nutrients. ${ }^{2}$ Apart from the low fat and high omega-3 and omega- 6 fatty acid contents, legumes present high content in proteins, dietary fibres, micronutrients (vitamins, minerals and amino acids) and antioxidants, such as phenolic compounds, (e.g. flavonoids, phenolic acids and tannins) with human health benefits, ${ }^{3,4}$ many of them present in highest concentrations, and/or in a more bioactive and bioavailable form, after the fermentation process. ${ }^{5}$ However, many of these fermented foods result from spontaneous fermentation processes, being traditionally produced by ancient techniques, which do not fully meet the quality standards and reproducibility demanded by modern-day consumers. Furthermore, some of these foods are poorly characterized regarding the effect of the fermentative process on their nutritional, structural and rheological properties. ${ }^{6}$

Miso is a Japanese fermented paste consumed in soups and as seasoning or flavouring agent. ${ }^{7,8}$ This fermented paste is usually produced using soybean, koji (rice, barley or soybean inoculated with Aspergillus oryzae), salt and water, inoculated with an old miso culture. ${ }^{9,10}$ In addition to $A$. oryzae, lactic acid bacteria and yeasts are also involved. ${ }^{11}$

In miso preparation, koji is the main source of hydrolytic enzymes (e.g. amylases, maltase, proteases and lipases), that are involved in the digestion of the main legume structural components. $^{11}$

The substrate used to produce koji defines the three main types of miso known: rice, barley and soybean. ${ }^{8}$ Rice miso is the most popular in Japan and is generally classified as salty or sweet, based on the salt concentration (12-14\% salt for salty and $5-7 \%$ salt for sweet), flavour and colour (white, light-coloured or red). ${ }^{7}$

As an alternative to soybean, other legumes can be used to produce miso and create new, diverse and locally produced

\footnotetext{
Correspondence to: $R$ Santos, LEAF (Linking Landscape Environment Agriculture and Food) Research Centre, Instituto Superior de Agronomia, Universidade de Lisboa, Tapada da Ajuda, 1349-017, Lisbon, Portugal. E-mail: rcsantos@isa.ulisboa.pt

LEAF (Linking Landscape Environment Agriculture and Food) Research Centre, Instituto Superior de Agronomia, Universidade de Lisboa, Lisbon, Portugal
} 
fermented products, with lower environmental impact and higher economic value. ${ }^{12,13}$

Grass pea (Lathyrus sativus L.) is a traditional Portuguese legume with important social and economic impact - it is part of the traditional heritage of dryland communities - and a huge effort has been made to reintegrate this legume into the Portuguese and Mediterranean diet. However, the presence of the neurotoxic amino acid $\beta$-ODAP ( $\beta$-oxalyl-diamino propionic acid), discovered in the early 1960 s and its correlation to lathyrism, a neurodegenerative disease, has hindered its consumption. ${ }^{14}$

Besides its cultural relevance, grass pea is a highly nutritious legume, with a role in the prevention of several diseases. ${ }^{15}$ Nutritionally, grass pea is a source of fibre, starch, phenolic compounds and pre-biotics. ${ }^{16,17}$ In comparison to soybean (Glycine max L.), grass pea has higher starch content ${ }^{18,19}$ and lower fat, fibre and protein concentration, however, the protein quality (essential amino acid composition) is similar in both legumes. ${ }^{17}$ These properties show that this traditional legume can be used as a substitute raw material of soybean in the production of fermented Eastern-like foods, with additional environmental and economic advantages, particularly to soybean-importing countries, such as Portugal. ${ }^{13,20,21}$ Additionally, the removal of $\beta$-ODAP neurotoxin to a considerable degree, has been reported in solid-state fermentations using fungal strains of Rhizopus oligosporus and A. oryzae, pointing fermentation as a way to improve the safety of grass pea products. $^{22,23}$

The aim of the present work was to develop and characterize two types of innovative misos (salty and sweet) using grass pea through Eastern fermentation methods. The assessment of physicochemical characteristics (chemical composition, colour, texture and linear viscoelastic behaviour) was used to monitor the evolution of misos over the fermentation/maturation process, in comparison to traditional miso (made from soybean). The new grass pea miso intends to contribute to the diversification of healthier, more nutritious and safe diets, and promote the consumption of this traditional legume often undervalued by consumers.

\section{MATERIAL AND METHODS}

\section{Raw materials}

Misos (salty and sweet) were prepared using soybean or grass pea, koji, non-pasteurized miso, sea salt and demineralized water. Koji was produced using blanched rice - 'Carolino' Rice: Portuguese Japonica variety of Oryza sativa L. and A. oryzae (from Vision Brewing Co., Nedlands, Australia). All raw materials were purchased in the local market, with the exception of grass pea that was supplied by Simões \& Ramos, Lda. (Portuguese local producer from Alvaiázere region).

\section{Koji production}

Aspergillus oryzae inoculum was prepared by surface inoculation of a spore suspension in solid YPD medium: $5 \mathrm{~g} \mathrm{~L}^{-1}$ of yeast extract, $10 \mathrm{~g} \mathrm{~L}^{-1}$ of peptone, $20 \mathrm{~g} \mathrm{~L}^{-1}$ of dextrose and $20 \mathrm{~g} \mathrm{~L}^{-1}$ of agar. Plates were incubated at $28{ }^{\circ} \mathrm{C}$ up to vigorous growth.

To produce koji, the blanched rice was washed and hydrated overnight in demineralized water at room temperature $\left(25^{\circ} \mathrm{C}\right)$. After draining, the rice was steam cooked using a Thermomix TM31 Food Processor (Vorwerk Elektrowerke GmbH \& Co. KG, Wuppertal, Germany) for $30 \mathrm{~min}$ at $100{ }^{\circ} \mathrm{C}$ and cooled to room temperature (the weight of cooked rice was 1.8 times its dry weight). A suspension of $A$. oryzae spores was prepared in sterile water and the spores quantified using a Haemocytometer
Neubauer Improved (Hirschmann Laborgeräte GmbH \& Co. KG, Eberstadt, Germany). Rice was inoculated with the spore suspension to obtain $1 \times 10^{6}$ spores $\mathrm{g}^{-1}$ of cooked rice, spread in a perforated aluminium tray, covered with cheesecloth and incubated at room temperature, under static conditions, with daily manual agitation, until a strong growth and sporulation were observed at the surface of the grains.

\section{Miso production}

Grass peas and soybeans were washed and soaked overnight in demineralized water (around $18 \mathrm{~h}$ ) at room temperature. After draining, the legumes were cooked at $120^{\circ} \mathrm{C}$, 1 atm for $20 \mathrm{~min}$ (the weight of cooked soybean and grass pea was 2.2 and 2.5 times their dry weight, respectively). To obtain a smooth paste the cooked legumes were crushed using a Thermomix TM31 Food Processor (Vorwerk Elektrowerke GmbH \& Co. KG, Wuppertal, Germany), increasing gradually the speed each 30 s until speed 5 was reached, and then remaining at speed 5 for $5 \mathrm{~min}$.

Salty miso and sweet miso produced using grass pea were prepared with the base from the procedures described by Inoue et al. ${ }^{24}$ and by Chiou et al., ${ }^{25}$ respectively. The two soybean misos were prepared by the same procedures and used as control. Salty misos were produced mixing $650 \mathrm{~mL} \mathrm{~L}^{-1}$ cooked legumes (grass pea or soybean), $200 \mathrm{~g} \mathrm{~L}^{-1} \mathrm{koji}, 120 \mathrm{~g} \mathrm{~L}^{-1}$ sea salt, $20 \mathrm{~mL} \mathrm{~L}^{-1}$ demineralized water and $10 \mathrm{~mL} \mathrm{~L}^{-1}$ non-pasteurized miso from a previous batch (seed miso). In the case of sweet misos, $500 \mathrm{~mL} \mathrm{~L}^{-1}$ of cooked legumes were mixed with $450 \mathrm{~g} \mathrm{~L}^{-1} \mathrm{koji}$ and $50 \mathrm{~g} \mathrm{~L}^{-1}$ sea salt. Raw materials were mixed until a uniform pasty texture was obtained, then the paste was packed tightly into cylindrical glass jars ( $7 \mathrm{~mm}$ diameter and $5 \mathrm{~cm}$ height) with walls and bottom previously coated with salt, leaving $1.5 \mathrm{~cm}$ of headspace, and covering the top of the paste with salt. The containers were closed and incubated at $30{ }^{\circ} \mathrm{C}$ for 4 months. Periodically (each month for salty misos and twice a month for sweet misos), two containers of each miso were opened, the salt from the surface was removed and the fermented product was analysed, in order to assess the evolution of the chemical composition of misos over time. Two additional containers, collected at the same time were used for colour, texture and viscoelastic linear behaviour analyses of misos.

\section{Chemical composition analysis}

For each miso, aliquots of 5 to $10 \mathrm{~g}$ were taken and dried using a Universal Oven (Memmert GmbH \& Co. KG, Schwabach, Germany), at $40{ }^{\circ} \mathrm{C}$ (in order to maximize the preservation of polysaccharides) until the weight stabilized (between 48 and $72 \mathrm{~h}$ ). Dry matter was calculated based on fresh and dry weight. Dried material was grounded using a ProfiCook KSW 1021 Coffee Grinder (Clatronic International GmbH, Kempen Germany) to particles $<0.5 \mathrm{~mm}$. Digestible starch was determined in $100 \mathrm{mg}$ of dry miso powder using a Total Starch Assay Kit (AA/AMG) (Megazyme Ltd, Wicklow, Ireland) following the manufacturer's instructions. The ethanolic extract (with low molecular weight polysaccharides) obtained in the first step of the procedure was used to determine glucose content by anthrone-sulphuric acid method. ${ }^{26}$ For evaluation of $\mathrm{pH}$, phenolic compounds and ascorbic acid content, $1 \mathrm{~g}$ of powder was dissolved in $5 \mathrm{~g}$ of sterile demineralized water. This extract was incubated in a IKA KS 125 Basic Shaker (LTF Labortechnik GmbH \& Co. KG, Wasserburg (Bodensee), Germany) for $10 \mathrm{~min}$ at room temperature with slight agitation and centrifuged using a Z $383 \mathrm{~K}$ Centrifuge (HERMLE Labortechnik $\mathrm{GmbH}$, Wehingen, Germany) at $18000 \times g$ for 5 min 
at room temperature. Analyses were performed on the supernatant of this extract. The $\mathrm{pH}$ values were measured using a potentiometer: 213 Microprocessor pH Meter (Hanna Instruments Inc., Woonsocket, RI, USA). Total soluble sugar content ( ${ }^{\circ}$ Brix) was assessed using a HI 96801 Digital Refractometer (Hanna Instruments Inc.) and soluble proteins quantified according to Bradford.$^{27}$ Ascorbic acid was determined by redox titration with DCPIP (2,6-Dichlorophenolindophenol) using diluted extract to minimize colour interferences. ${ }^{28}$ The phenolic compounds concentration in the aqueous extract was determined using a CE1011 Spectrophotometer (Cecil Instruments Ltd, Cambridge, UK). The absorbance was measured at $280 \mathrm{~nm}$, a solution of gallic acid $^{29}$ was used as the calibration standard and the absorbance at $260 \mathrm{~nm}$ was taken in consideration to overcome the possible interference of nucleic acids.

\section{Colour analysis}

The colour of misos was instrumentally evaluated using a tristimulus colorimeter: CR-400 Chroma Meter (Konica Minolta Inc., Tokyo, Japan), with standard illuminant D65 and a visual angle of $2^{\circ}$. Colour parameters $\left(L^{*}, a^{*}\right.$ and $\left.b^{*}\right)$ were determined by the CIELab system. ${ }^{30}$ The colour evolution of misos was evaluated according to $\Delta E^{*}$ parameter, which can be correlated with the total colour difference between two samples. This parameter is designated by the imaginary line distance between sample and standard, and it is calculated based on the $\Delta L^{*}, \Delta a^{*}, \Delta b^{*}{ }^{30}$

$$
\Delta E_{a b}^{*}=\sqrt{\left(L_{2}^{*}-L_{1}^{*}\right)^{2}+\left(a_{2}^{*}-a_{1}^{*}\right)^{2}+\left(b_{2}^{*}-b_{1}^{*}\right)^{2}}
$$

The analyses were carried out directly in the miso containers at $20 \pm 1{ }^{\circ} \mathrm{C}$, using a white standard $\left(L^{*}=97.21 ; a^{*}=0.14 ; b^{*}=1.99\right)$ and four replicates per sample were performed.

\section{Texture measurements - firmness and adhesiveness}

The texture analysis of misos was performed using a TA.XTplus Texture Analyser (Stable Micro-Systems, Godalming, UK) in a temperature-controlled room at $20 \pm 1{ }^{\circ} \mathrm{C}$. The results of the texture of the misos were expressed in terms of firmness and adhesiveness obtained by texture profile analysis (TPA) in penetration tests - 'two bites' - with a cylindrical probe of $11 \mathrm{~mm}$ diameter: $15 \mathrm{~mm}$ penetration, $2 \mathrm{~mm} \mathrm{~s}^{-1}$ pre- and posttest speed, $1 \mathrm{~mm} \mathrm{~s}^{-1}$ test speed, $5 \mathrm{~s}$ of waiting time, and with a load cell of $5 \mathrm{~kg}$. Miso samples were analysed directly in their own containers ( $7 \mathrm{~mm}$ diameter and $5 \mathrm{~cm}$ height), performing at least four replicates per sample.

Firmness $(\mathrm{N})$ is the maximum force recorded in texturogram (force versus time) in first penetration cycle and represents the force required to compress the material between the molars. Adhesiveness (N.s.) is the negative area of the texturogram, and represents the work required to remove the probe from the material, and related with the force required to remove food sticking to mouth during chewing.

\section{Small amplitude oscillatory measurements}

The study of the viscoelastic linear behaviour of misos was performed by dynamic small-amplitude oscillatory shear (SAOS) measurements in a controlled stress rheometer: Thermo Scientific HAAKE MARS III (ThermoFisher Scientific, Karlsruhe, Germany), with an UTC - Peltier system for temperature control. A serrated parallel plate sensor system (PP20-20 mm diameter) was used to overcome the slip effect, with a $1.5 \mathrm{~mm}$ gap, previously optimized for this material.

For each sample, stress sweep tests (variation of the sinusoidal stress applied to a constant frequency value) were applied to define the viscoelastic linear region - the range of stress in which the viscoelastic moduli ( $G$ ', storage modulus and $G^{\prime \prime}$, loss modulus) are independent of the applied stress. The mechanical spectrum of each miso was obtained by frequency sweep tests: variations of $G^{\prime}$ and $G^{\prime \prime}$ over a range of different frequencies at a constant stress, within the linear viscoelastic region.

The samples of misos were placed on the measuring device, covered with paraffin oil to avoid evaporation and stabilized for $10 \mathrm{~min}$ at $20^{\circ} \mathrm{C}$. Frequency sweep tests were conducted, after 10 min of sample stabilization, by varying the frequency between 0.001 and $100.0 \mathrm{~Hz}$ at $20^{\circ} \mathrm{C}$ with a constant shear stress inside the linear viscoelastic region (previously determined by a stress sweep test: $f=0.1 \mathrm{~Hz}$ and $\tau=0.1-100 \mathrm{~Pa}$ ). The two tests were performed at least in triplicate.

\section{Statistical analysis}

The mean and standard deviation of the experimental data and statistical analysis were conducted by GraphPad Prism software (version 5.0). One way analysis of variance (ANOVA) was performed and when significant differences were found between treatments, post hoc analysis using Tukey's test was conducted $(\alpha=0.05)$.

\section{RESULTS AND DISCUSSION}

\section{Chemical composition analysis}

Specific nutritional compounds of the four misos produced were analysed (Table 1). The results obtained are in agreement with the nutritional values of grass pea and soybean, mentioned in the literature. ${ }^{18,19}$ As expected, the two types of miso from grass pea presented higher content in carbohydrates - digestible starch and glucose - than misos produced using soybean, both at the beginning of fermentation and after 4 months (120 days).

Although the fermentation process is supposed to lead to degradation of certain nutrients, the content of digestible starch occasionally increased over time. This may be related to the breakdown of inter-molecular bonds resulting from protein hydrolysis, which causes a lower degree of structuring and greater accessibility of starch to digestive enzymes. ${ }^{31}$ The results are in agreement with this theory, since soluble proteins were barely detectable in the different misos produced (data not shown).

Regarding the antioxidant compounds, the highest values of ascorbic acid were observed in both soybean misos. In the case of phenolic compounds, the initial values correspond to the concentration in the aqueous extract obtained for the paste after soaking, dehulling and cooking. During these processes, most of the soluble phenols were extracted/lost to the water, which may justify the low concentration obtained in comparison to those described for the vegetable raw materials. Nevertheless, within the four misos, grass pea and soybean sweet misos presented similar values, that were slightly lower in both salty misos. As the fermentation/maturation proceeds, the content of ascorbic acid and phenolic compounds increased for all misos (grass pea miso presented the highest rise), possibly as an effect of yeast and/or lactic acid bacteria present in the misos. ${ }^{32}$ The increase in such health-beneficial compounds over the fermentation process, was also reported by Shin et al., ${ }^{33}$ indicating fermentation as a process to add value to legumes. As for ${ }^{\circ}$ Brix, all misos 
Table 1. Chemical composition of the four misos developed at the beginning ( 0 days) and after 4 months (120 days) of fermentation/maturation process

\begin{tabular}{|c|c|c|c|c|c|c|c|c|c|}
\hline \multirow{2}{*}{$\begin{array}{l}\text { Time } \\
\text { (days) }\end{array}$} & \multicolumn{2}{|c|}{ Misos } & \multirow{2}{*}{$\begin{array}{l}\text { Dry matter } \\
\left(\mathrm{g} \mathrm{kg}^{-1}\right)\end{array}$} & \multirow{2}{*}{$\begin{array}{c}\text { Digestible } \\
\text { starch }\left(\mathrm{g} \mathrm{kg}^{-1}\right)\end{array}$} & \multirow{2}{*}{$\begin{array}{l}\text { Glucose } \\
\left(\mathrm{g} \mathrm{kg}^{-1}\right)\end{array}$} & \multirow{2}{*}{$\begin{array}{c}\text { Ascorbic } \\
\text { acid }\left(\mathrm{mg} \mathrm{kg}^{-1}\right)\end{array}$} & \multirow{2}{*}{$\begin{array}{l}\text { Phenolic compounds } \\
\text { in aqueous extract } \\
\qquad\left(\mathrm{mg} \mathrm{kg}^{-1}\right)\end{array}$} & \multirow[b]{2}{*}{${ }^{\circ}$ Brix } & \multirow[b]{2}{*}{$\mathrm{pH}$} \\
\hline & Type & Legume & & & & & & & \\
\hline \multirow[t]{4}{*}{0} & Salty & Grass pea & 458.1 & 58.1 & 144.0 & 12.9 & 0.2 & 45.6 & 5.5 \\
\hline & & Soybean & 491.1 & 13.3 & 21.3 & 36.4 & 0.4 & 45.6 & 5.5 \\
\hline & Sweet & Grass pea & 591.5 & 85.5 & 231.2 & 6.0 & 0.5 & 44.4 & 5.0 \\
\hline & & Soybean & 601.1 & 63.5 & 220.7 & 70.6 & 0.6 & 46.2 & 4.8 \\
\hline \multirow[t]{4}{*}{120} & Salty & Grass pea & 536.5 & 56.5 & 194.2 & 62.6 & 0.5 & 46.2 & 4.8 \\
\hline & & Soybean & 486.0 & 18.8 & 129.7 & 102.2 & 0.7 & 46.8 & 5.0 \\
\hline & Sweet & Grass pea & 639.9 & 102.9 & 226.7 & 169.1 & 1.6 & 43.8 & 4.2 \\
\hline & & Soybean & 553.1 & 56.5 & 177.3 & 227.5 & 1.5 & 45.6 & 4.5 \\
\hline
\end{tabular}

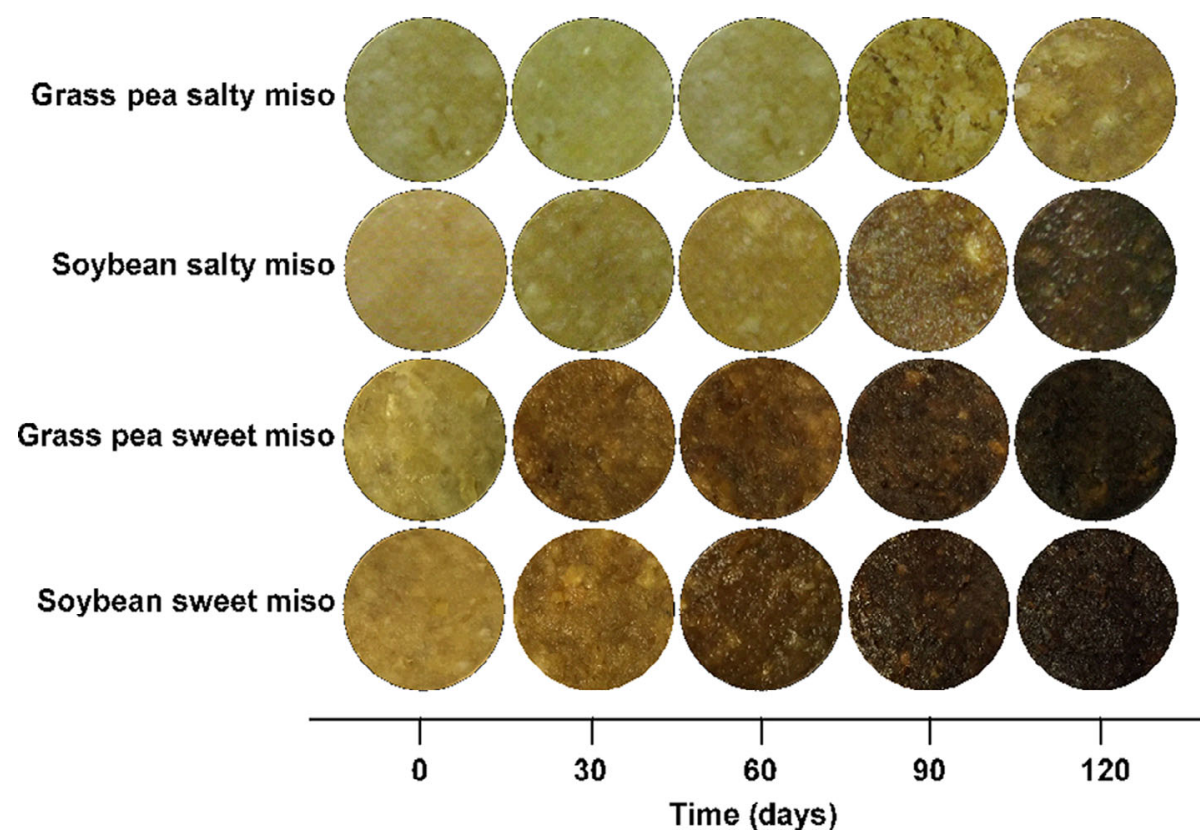

Figure 1. Colour evolution of the misos over 4 months (120 days) of fermentation/maturation process.

presented similar values that remained somehow constant along the fermentation/maturation period.

\section{Colour analysis}

Throughout the fermentation/maturation process, the colour of the fermented legume paste changed, darkening in all cases (Fig. 1). Both salty misos showed an identical evolution from the beginning of fermentation until after 30 days. Afterwards, soybean misos showed a faster colour evolution, and consequently a final darker colour. In sweet misos the reverse was observed, with faster darkening of grass pea misos, presenting a darker colour after the first month of fermentation. Still, after 120 days, all misos presented a similar red dark colour, except grass pea salty miso that was lighter.

The evaluation of the colour of the misos was also followed by $\Delta E^{*}$, which quantifies the colour difference between a sample and a standard. The $\Delta E^{*}$ value at the beginning of fermentation (day 0 ) was used as standard colour for each sample. The evolution of $\Delta E^{*}$ value (Fig. 2) is in accordance with the visual colour observation through the fermentation process (Fig. 1).

\section{Colour}

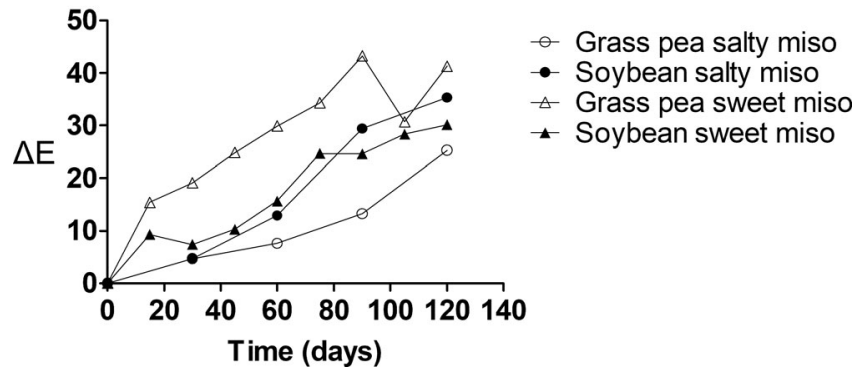

Figure 2. Colour evolution of the misos expressed in terms of $\Delta E^{*}$ values over 4 months (120 days) of fermentation/maturation process.

The browning of all misos may result from the enzymatic oxidation by polyphenol oxidase (PPO) and/or auto-oxidation involving phenolic pigmented compounds, such as flavones, and from reactions between reducing sugars and amino acids (Maillard reactions). ${ }^{34,35}$ Besides, the production of brown compounds, such 
as soyamelanins (insoluble brown polymers from soybeans), was also reported. ${ }^{11}$ The fast evolution of the grass pea sweet miso and its final darkest colour may reflect its initial high content in glucose and phenols, favouring Maillard reactions and oxidation reactions of phenolic compounds.

On the contrary, grass pea salty miso presented the slowest evolution and the lightest colour. Besides the effect of salt in the control of microbial growth and activity, ${ }^{36}$ ensuring the quality and safety of food, this compound has also a potential PPO inhibitory effect, ${ }^{37}$ leading to a great inhibition of the enzymatic browning as was observed in the two salty misos. Additionally, the lower phenolic content of grass pea salty miso has probably contributed to fewer oxidation reactions and consequently to the lighter colour of salty misos.

\section{Texture measurements - firmness and adhesiveness}

The evolution of the texture of all misos was evaluated through a TPA and the results of firmness and adhesiveness are presented in Fig. 3.

The data in Fig. 3(a) showed that the firmness of both sweet misos decreased dramatically after 15 days, from $51.63 \mathrm{~N}$ to $6.52 \mathrm{~N}$ in grass pea miso and from $26.46 \mathrm{~N}$ to $2.55 \mathrm{~N}$ in soybean sweet miso, remaining then constant until 120 days of fermentation/maturation ( $\alpha=0.05$ ). A different behaviour was noticed for salty misos, which began with a much lower firmness that decreased significantly after 30 days (from $8.87 \mathrm{~N}$ to $4.02 \mathrm{~N}$ for grass pea miso and from $4.62 \mathrm{~N}$ to $2.68 \mathrm{~N}$ for soybean miso) and stabilized in the following 3 months.

In relation to adhesiveness (Fig. 3(b)), in the first 15 days, soybean sweet miso presented the most evident reduction (from $27.76 \mathrm{~N}$ to $3.11 \mathrm{~N}$ ) compared to grass pea sweet miso (from $11.63 \mathrm{~N}$ to $2.73 \mathrm{~N}$ ). The adhesiveness reduction in salty misos was not significant $(\alpha=0.05)$.

The more pronounced reduction of texture parameters in sweet misos may be attributed to the higher concentration of koji and therefore to the higher enzymatic content, which is responsible for the larger degradation of its structural components. ${ }^{11,38}$ According to Hutkins, approximately $50 \%$ of the total protein and $75 \%$ of the polysaccharides are completely hydrolysed in the course of the fermentation. ${ }^{8}$ Furthermore, starch-bound proteins (around 0.6\%), have an important role in the high food firmness since they avoid starch digestion and consequently the increase of digestible starch. ${ }^{39}$

(a)

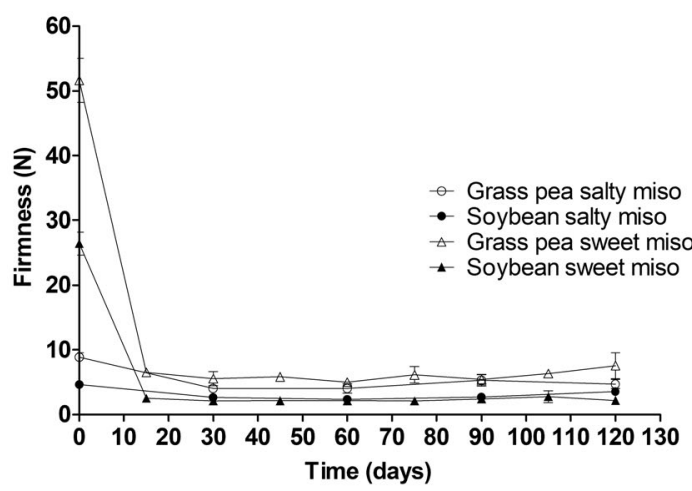

The fact that sweet misos have more hydrolytic enzymes and higher carbohydrates content (at the beginning of the fermentation) than salty misos, possibly led to a larger reduction of the more structured starch and of glucose, and consequently, a larger decrease in firmness and adhesiveness as compared to salty misos, which is in accordance to the observations of several authors. ${ }^{40,41}$

The higher carbohydrates and lower protein content of grass pea ${ }^{18,19}$ can also explain the differences observed between salty and sweet misos from grass pea and soybean. In particular, the differences in these compounds may influence directly the higher initial firmness and adhesiveness and the more evident reduction of firmness and adhesiveness observed for grass pea misos in the first 15 days (sweet miso) and 30 days (salty miso). Furthermore, in the last 3 months of fermentation, firmness and adhesiveness remained almost constant ( $\alpha=0.05$ ). During this period, nonphysical changes are predominant, such as: amino acids production, fatty acids esterification and some degradation of sugar compounds, which are responsible for organoleptic properties, which may explain their evolution in misos. ${ }^{11}$

\section{Small amplitude oscillatory measurements}

Comparatively to texture profiles, the viscoelastic behaviour characterization allowed a more detailed analysis of the evolution of the internal structure of the different misos throughout the fermentation/maturation time. The mechanical spectra obtained from the frequency sweep tests are shown in Fig. 4.

The storage modulus $\left(G^{\prime}\right)$ and the loss modulus $(G ")$ decreased over time in all misos, indicating a reduction in their degree of structuring. However, grass pea misos (salty and sweet) presented the highest values of $G^{\prime}$ and $G^{\prime \prime}$, which may reflect a stronger internal structure system, compared to soybean misos, due to the higher starch content of grass pea misos. These results are in agreement with the firmness values and suggest the important role of koji in the fermentation process of miso, as the main source of hydrolytic enzymes., 8,11 The enzymatically digested and fermented products from grass pea/soybeans have lower ability to form inter-molecular linkages and aggregates, ${ }^{42}$ influencing the structural properties of the final product and decreasing viscoelastic functions.

In all the mechanical spectra $G$ ' values are higher values than $G$ " (predominance of the elastic component) and there is a similar distance between the two viscoelastic functions. The evolution (b)

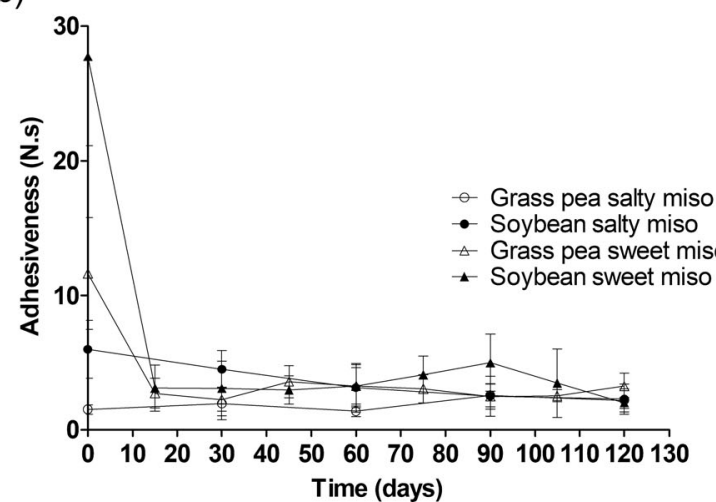

Figure 3. Evolution of the texture parameters of the misos over 4 months ( 120 days) of fermentation/maturation process. (a) Mean firmness values, (b) Mean adhesiveness values. 
(a)

Grass pea salty miso
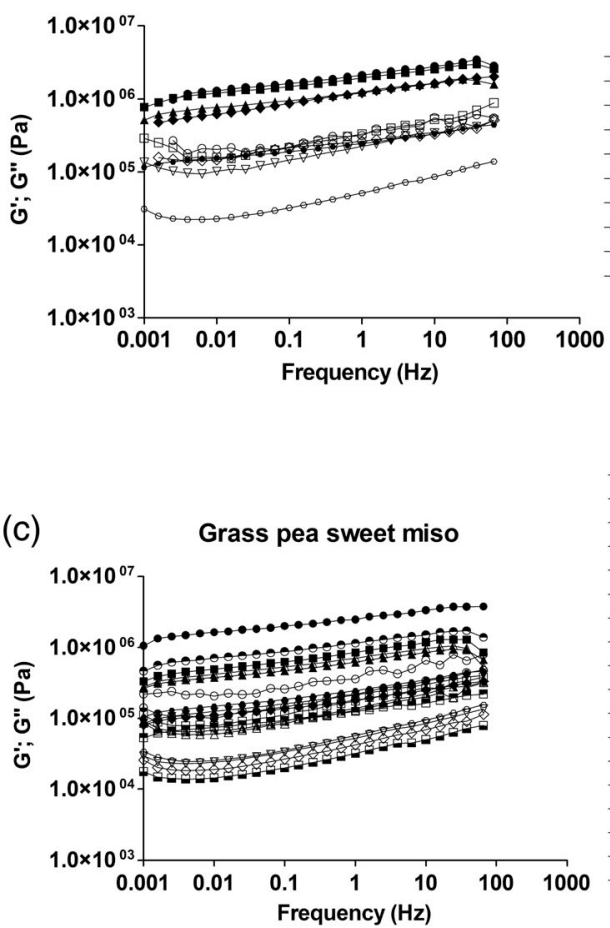

(b)

Soybean salty miso

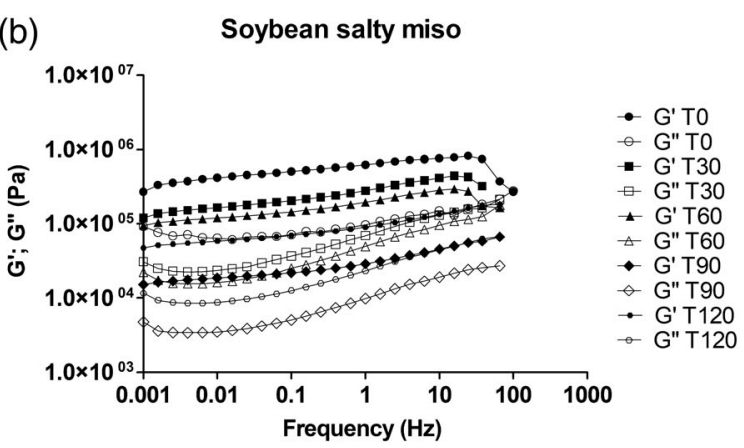

- G'TO $^{\prime}$

- G" T0

+ G' T15

- G'T30

* G" T30

- G' T45

- G" T45

- G" T60

$*$
$*$

- G' T90

$\diamond$ G" T90

- G' T105

- G" T105

- G" T105

- $G^{\prime} T 120$

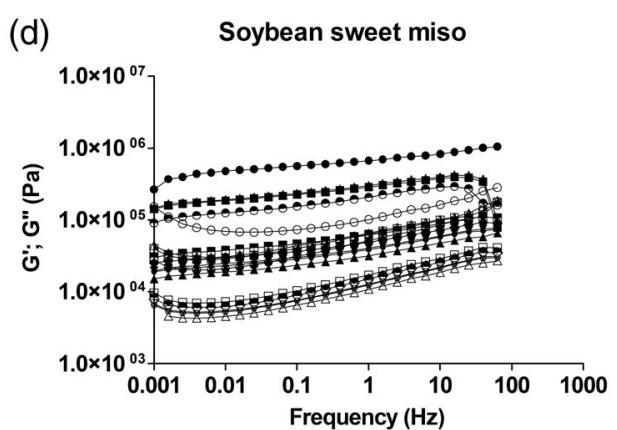

- G'TO $^{\prime}$

- G" T0

- G' T15

- G" T15

- G' T30

- G" T30

- G'T45

- G" T45

- G' T60

G" T60

- G"T60

- G' T75

- G' T90

$\diamond$ G" T90

- G'T105

- G" T105

- G'120

- G" 120

Figure 4. Evolution of the viscoelastic moduli ( $G$ and $G^{\prime \prime}$ ) over 4 months ( 120 days) of fermentation/maturation process. (a) Mechanical spectra of the grass pea salty miso, (b) Mechanical spectra of the soybean salty miso, (c) Mechanical spectra of the grass pea sweet miso, (d) Mechanical spectra of the soybean sweet miso.

(a)

Salty misos

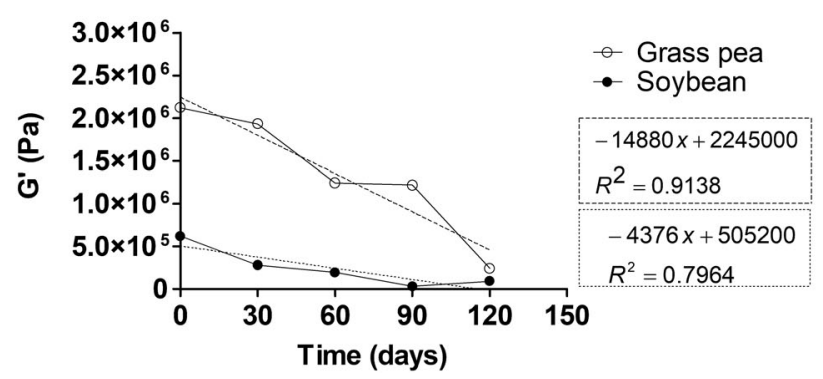

(b)

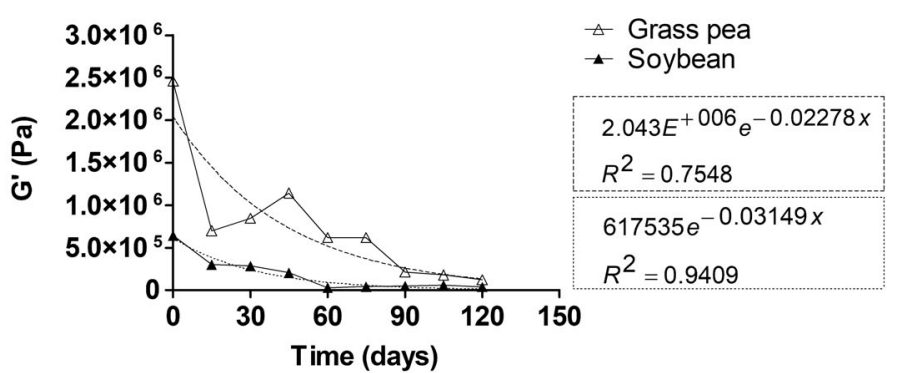

Figure 5. Evolution of $G^{\prime}$ modulus at $1 \mathrm{~Hz}$ frequency over 4 months (120 days) of fermentation/maturation process. (a) Grass pea and soybean salty miso values, (b) Grass pea and soybean sweet miso values.

of $G^{\prime}$ and $G^{\prime \prime}$ have a slight dependence with oscillation frequency, for all misos, suggesting a similar structure with a weak-gel like behaviour.

In general, all misos presented a similar behaviour over the 4 months of fermentation (reduction of the viscoelastic moduli), reflecting identical microbiological and structural phenomena similar internal structure.

In order to obtain a more detailed analysis about the variation of the viscoelastic behaviour, the evolution of $G^{\prime}$ obtained at $1 \mathrm{~Hz}$ is summarized in Fig. 5. As expected, grass pea misos presented higher initial values of $G^{\prime} 1 \mathrm{~Hz}$, associated with a more structured material, and a more abrupt decrease over the fermentation/maturation process. In the first 30 days, viscoelastic moduli reduction was more pronounced for grass pea sweet miso showing an exponential decay (from 2.46 MPa to 0.85 MPa and from 2.12 MPa to $1.93 \mathrm{MPa}$ for sweet and salty grass pea miso, respectively).

The fact that grass pea misos have a higher starch content than soybean misos may lead to a greater impact of degradation over time. The proteolytic enzymatic action of koji ${ }^{43}$ may have contributed to the availability of non-digestible starch for degradation, promoting a faster kinetic of maturation. However, soybean misos G' $1 \mathrm{~Hz}$ values were the first to stabilize (after 90 and 60 days for salty and sweet miso, respectively), indicating an earlier matured state as was observed by the apparent colour evolution of misos (Fig. 1). This can be explained by the lower starch content present in soybean that may have been degraded (by koji enzymes) before grass pea misos, reaching sooner the final values for $G$ '. Apart from that, the higher ascorbic acid content leads to higher 
antioxidant activity, inhibiting oxidation reactions and contributing to the structure stabilization. ${ }^{44}$

\section{CONCLUSIONS}

In this study, two types of innovative grass pea misos were produced (sweet and salty) and compared to the standard miso made from soybean, used as a control. The analyses of chemical composition, colour, texture and linear viscoelastic behaviour of these new misos, allowed to monitor the evolution of the fermentation/maturation process and obtain a deeper knowledge of physicochemical changes occurring throughout this process.

The results revealed that the composition of raw materials and the formulation of the misos have an important impact on the evolution of firmness, internal structure system, colour, and maturation kinetics over the fermentation process. However, after 120 days, the texture and internal structuring system of grass pea misos were similar to soybean misos, demonstrating that grass pea can be used as a raw material to produce a new miso with physicochemical characteristics equivalent to traditional miso.

Sensorial analysis and consumers' acceptance of grass pea miso are being performed (Rocha et al., unpublished data, 2019). Preliminary results obtained so far point to a high degree of consumer acceptance of the new grass pea miso. Based on these results, it is expected that grass pea miso will contribute to increase the food diversity and legume consumption, while promoting the environmentally sustainable and local production of this neglected and traditional crop.

\section{ACKNOWLEGEMENTS}

This work was supported by Fundação para a Ciência e a Tecnologia (Portugal) through the research unit UID/AGR/04129/2013 (LEAF) and the project QUALATY (PTDC/AGR-TEC/0992/2014).

\section{REFERENCES}

1 Baumung R and Hoffmann I, Animal genetic diversuty and sustainable diets, in International Scientific Symposium - Biodiversity and Sustainable Diets United Against Hunger, ed. by Burlingame B and Dernini S. Division NaCP and FAO. Food and Agriculture Organization of the United Nations (FAO), Rome, pp. 82-92 (2012).

2 Granato D, Branco GF, Nazzaro F, Cruz AG and Faria JA, Functional foods and nondairy probiotic food development: trends, concepts, and products. Compr Rev Food Sci Food Saf 9:292-302 (2010).

3 Watanabe $\mathrm{H}$, Beneficial biological effects of miso with reference to radiation injury, cancer and hypertension. J Toxicol Pathol 26: 91-103 (2013).

4 Omoni $\mathrm{AO}$ and Aluko RE, Soybean foods and their benefits: potential mechanisms of action. Nutr Rev 63:272-283 (2005).

5 Messina MJ, Legumes and soybeans: overview of their nutritional profiles and health effects. Am J Clin Nutr 70:439s-450s (1999).

6 Handoyo T and Morita N, Structural and functional properties of fermented soybean (tempeh) by using Rhizopus oligosporus. Int J Food Prop 9:347-355 (2006).

7 Kiuchi K, Miso \& Natto. Food Culture 3:7-10 (2001).

8 Hutkins RW, in Fermentation of foods in the Orient, in Microbiology and Technology of Fermented Foods, ed. by Hutkins RW. Blackwell Publishing, lowa City, IA, pp. 419-455 (2006).

9 Mullin WJ, Miso as a functional food, in Asian Functional Foods, ed. by Shi J, Ho C-T and Shahidi F. CRC Press - Taylor \& Francis, New York, pp. 537-553 (2005).

10 Mani $V$ and Ming LC, Tempeh and other fermented soybean products rich in isoflavones, in Fermented Foods in Health and Disease Prevention, ed. by Frias J, Martinez-Villaluenga $C$ and Peñas E. Academic Press, Boston, MA, pp. 453-474 (2017).

11 Shibasaki K and Hessbltine C, Miso fermentation. Econ Bot 16:180-195 (1962).
12 Prista C, When traditional Western crops meet Eastern microbial eukaryotic fermenters for the production of innovative "gourmet" food. Legume Perspect. 9:14-15 (2015).

13 Abiose SH, Allan MC and Wood BJB, Microbiology and biochemistry of miso (soy paste) fermentation, in Advances in Applied Microbiology, ed. by Laskin Al. Academic Press, New York, pp. 239-265 (1982).

14 Hailu D, Abera S and Teka TA, Effects of processing on nutritional composition and anti-nutritional factors of grass pea (Lathyrus sativus L.): a review. Food Sci Qual Manage 36:61-70 (2015).

15 Rao S, A look at the brighter facets of $\beta$-N-oxalyl-L- $\alpha$, $\beta$-diaminopropionic acid, homoarginine and the grass pea. Food Chem Toxicol 49:620-622 (2011).

16 Gupta P, Udupa SM, Gupta DS, Kumar J and Kumar S, Population structure analysis and determination of neurotoxin content in a set of grass pea (Lathyrus sativus L.) accessions of Bangladesh origin. Crop J 6:435-442 (2018).

17 Ferranti $\mathrm{P}$, Preservation of food raw materials, in Reference Module in Food Sciences, Amsterdam: Elsevier, pp. 1-3 (2016).

18 Rotter R, Marquardt R and Campbell $C$, The nutritional value of low lathyrogenic Lathyrus (Lathyrus sativus) for growing chicks. Br Poultry Sci 32:1055-1067 (1991).

19 Banaszkiewicz T, Nutritional value of soybean meal, in Soybean and Nutrition, ed. by El-Shemy HA. InTech, Rijeka, pp. 1-20 (2011).

20 European Commission, Oilseeds and protein crops statistics. [Online]. European Union (2019). Available: https://ec.europa.eu/info/foodfarming-fisheries/farming/facts-and-figures/markets/overviews/ market-observatories/crops/oilseeds-and-protein-crops_ en\#production [20 February 2019].

21 Portal EUOD, Trade monitoring through customs surveillance data. [Online]. European Union (2019). Available: https://data.europa.eu/ euodp/en/data/dataset/oilseeds-trade/resource/3f7f2c8e-99054fb6-8280-de7cce0602a8 [20 February 2019].

22 Lambein F, Diasolua Ngudi D and Kuo Y-H, Progress in prevention of toxico-nutritional neurodegenerations. Afr Technol Dev Forum J 6: 60-65 (2010).

23 Yigzaw Y, Gorton L, Solomon T and Akalu G, Fermentation of seeds of Teff (Eragrostis teff), grass-pea (Lathyrus sativus), and their mixtures: aspects of nutrition and food safety. J Agric Food Chem 52: 1163-1169 (2004).

24 Inoue K, Gotou T, Kitajima H, Mizuno S, Nakazawa T and Yamamoto N, Release of antihypertensive peptides in miso paste during its fermentation, by the addition of casein. J Biosci Bioeng 108:111-115 (2009).

25 Chiou RYY, Ferng S and Beuchat LR, Fermentation of low-salt miso as affected by supplementation with ethanol. Int J Food Microbiol 48: 11-20 (1999).

26 De Bruyn J, Van Keulen $\mathrm{H}$ and Ferguson J, Rapid method for the simultaneous determination of glucose and fructose using anthrone reagent. J Sci Food Agric 19:597-601 (1968).

27 Bradford MM, A rapid and sensitive method for the quantitation of microgram quantities of protein utilizing the principle of proteindye binding. Anal Biochem 72:248-254 (1976).

28 Oliveira R, Godoy $\mathrm{H}$ and Prado $\mathrm{M}$, Optimization of a colorimetric method to determine ascorbic acids in fruit jelly. Ciência e Tecnologia de Alimentos 30:244-249 (2010).

29 Waterhouse A, Determination of total phenolics. Curr Protocol Food Anal Chem 6:1.1.1-1.1.8 (2002).

30 Sahin S and Sumnu S, Electromagnetic properties, in Physical Properties of Foods, ed. by Sahin S and Sumnu S. Springer Science \& Business Media, New York, pp. 157-189 (2006).

31 Elkhalifa AEO, Schiffler B and Bernhard R, Effect of fermentation on the starch digestibility, resistant starch and some physicochemical properties of sorghum flour. Food Nahrung 48:91-94 (2004).

32 Katina K, Laitila A, Juvonen R, Liukkonen K-H, Kariluoto S, Piironen V et al., Bran fermentation as a means to enhance technological properties and bioactivity of rye. Food Microbiol 24:175-186 (2007).

33 Shin H-Y, Kim S-M, Lee JH and Lim S-T, Solid-state fermentation of black rice bran with Aspergillus awamori and Aspergillus oryzae: effects on phenolic acid composition and antioxidant activity of bran extracts. Food Chem 272:235-241 (2019).

34 Martinez MV and Whitaker JR, The biochemistry and control of enzymatic browning. Trends Food Sci Technol 6:195-200 (1995).

35 Baltes $W$, Chemical changes in food by the maillard reaction. Food Chem 9:59-73 (1982). 
36 Guinee TP and Fox PF, Salt in cheese: physical, chemical and biological aspects, in Cheese: Chemistry, Physics and Microbiology, ed. by Fox PF, PL MS, Cogan TM and Guinee TP. Elsevier, London, pp. 207-260 (2004).

37 Piližota V and Šubarić D, Enzymatic browning of foods. Food Technology and Biotechnology 36:219-227 (1998).

38 McKenna BM, Introduction, in Texture in Food, ed. by by McKenna BM. Woodhead Publishing, Cambridge, pp. xv-xvi (2003).

39 Alcázar-Alay SC and Meireles MAA, Physicochemical properties, modifications and applications of starches from different botanical sources. Food Sci Technol 35:215-236 (2015).

40 Adhikari B, Howes T, Bhandari BR and Truong V, Stickiness in foods: a review of mechanisms and test methods. Int J Food Prop 4:1-33 (2001).
41 Y-y X, J-f K, Qi X-n, Ye Y-j, Wu Z-X, J-y C et al., A comprehensive investigation of starch degradation process and identification of a transcriptional activator MabHLH 6 during banana fruit ripening. Plant Biotechnol J 16:151-164 (2018).

42 Wilson LA, Soy foods, in Practical Handbook of Soybean Processing and Utilization, ed. by Erickson DR. AOCS Press and the United Soybean Board, Urbana, IL, pp. 428-459 (1995).

43 Elfalleh W, Sun C, He S, Kong B and Ma Y, Changes in enzymatic activities during "koji" incubation and natural fermentation of soybean paste. J Food Process Preserv 41:e13302 (2017).

44 Cort WM, Antioxidant properties of ascorbic acid in foods, in Ascorbic Acid: Chemistry, Metabolism, and Uses, ed. by Seib PA and Tolbert BM. American Chemical Society, Washington, DC, pp. 533-550 (1982). 